\title{
Uso de lodos residuales como fertilizante en eucalipto - diagnóstico de investigación
}

\author{
Use of residual sludge as a fertilizer on eucaliptus - research diagnosis \\ Alessandro Reinaldo Zabottoํ, Enrique Alonso Zuñiga ${ }^{3}$, Luz María Ruiz Machuca ${ }^{3}$, \\ Fernando Broetto ${ }^{2}$, Armando Reis Tavares ${ }^{1}$, Shoey Kanashiro ${ }^{1}$
}

\begin{abstract}
RESUMEN
El lodo residual es un residuo contaminante, proveniente del proceso de tratamiento de residuos urbanos. Su disposición final puede tornarse un problema al ser descartado de manera incorrecta, generando graves problemas ambientales y salubres. Sin embargo, por poseer alto contenido de materia orgánica, macro e micronutrientes, puede ser aprovechado en plantaciones forestales como suplemento fertilizante o mejorador del suelo, siendo una alternativa interesante para su destino final y reciclaje. La silvicultura, se caracteriza por aportar materia prima para el desarrollo de diversos productos, teniendo como base los bosques plantados. En este contexto, el cultivo de eucalipto se destaca debido a la gran importancia para la economía brasileña, teniendo inclusive aumento expresivo en su plantación en las últimas décadas. Por lo tanto, debido a los problemas ambientales que pueden ser causados por la colocación del lodo residual en el medio ambiente, este trabajo tiene como objetivo diagnosticar el estado del arte de la utilización del lodo residual en el cultivo de eucalipto.
\end{abstract}

Palabras clave: eucalipto, nutrición vegetal, silvicultura.

The residual sludge is a contaminating waste, coming from the urban waste treatment process. Its final disposal can become a problem when it is discarded incorrectly, generating serious environmental and health problems. However, it has a high content of organic matter, macro and micronutrients, and can be used in forest plantations as a fertilizer supplement or soil conditioner, being an interesting alternative to its final destination and recycling. Forestry is characterized by providing raw materials for the development of various products based on planted forests. In this context, the eucalyptus crop stands out due to the great importance for Brazilian economy, with an expressive increase in its plantation area in the last decades. Therefore, due to the environmental problems that can be caused by the deposit of residual sludge in the environment, this work aims to diagnose the state of the art of the use of residual sludge in the eucalyptus crop.

Key words: eucalyptus, plant nutrition, forestry.

\section{Introducción}

El volumen de lodo producido por grandes centros urbanos, demanda grandes áreas para su disposición y utilización en zonas reforestadas, destacándose especialmente el eucalipto.

En Brasil, diversos estudios con el uso del lodo han sido realizados en las últimas tres décadas, evaluando su aplicación en varios cultivos hortícolas, así como en especies forestales. Algunas de las ventajas del uso del lodo para fines agrícolas son: su reciclaje y aplicación al suelo reincorporando los nutrientes que fueron extraídos por los diferentes cultivos (Ley de la restitución).

El lodo cuando es descartado de forma inadecuada, puede contaminar al medio ambiente y causar riesgos a la población. La disposición final en rellenos sanitarios, conlleva a elevar los costos de tratamiento para su reciclaje y producción final. El proceso de tratamiento del lodo y la disposición final de este pueden alcanzar hasta el $50 \%$ de los costos operacionales de las estaciones

\footnotetext{
Instituto de Botánica. San Pablo, SP.

Departamento de Química y Bioquímica, Instituto de Biociencias. Universidad Estatal de San Pablo. Botucatu, SP.

Facultad de Ciencias Agronómicas, Universidad Estatal de San Pablo. Botucatu, SP.

* Autor por correspondencia: alezabotto@gmail.com; broetto@ibb.unesp.br
} 
de tratamiento: por lo cual existen alternativas para el reciclado de este residuo, siendo la agricultura y la actividad forestal las formas más viables de reciclado (Bettiol y Camargo, 2000). El residuo, después de haber sido tratado, puede ser utilizado como fertilizante orgánico en la recuperación de áreas degradas e en áreas de silvicultura (Zabotto et al., 2018).

El uso del lodo como fertilizante en zonas reforestadas es viable y prometedor, despertando gran interés agronómico, considerándose como una alternativa económica viable para la substitución de fertilizantes minerales, en especial los nitrogenados, además del aumento en la producción de madera (Poggiani y Silva, 2005). Su utilización en la agricultura como fertilizantes o como mejorador del suelo no causa daños a la población, siendo está la opción más sustentable para el reciclaje de lodo (Clarke y Smith, 2011). Esta práctica puede evitar altos costos para su destino final, reduciendo significativamente el impacto para el medio ambiente y para la sociedad (Andrade et al., 2018).

El uso del lodo como fertilizante, puede presentar beneficios para las plantas. Sin embargo, para su uso es necesario realizar análisis químicos y físicos, en función de la variación del contenido de macro y micronutrientes, además del monitoreo de los niveles de metales pesados que se pueden acumular en la cadena alimenticia (Oliveira et al., 2018).

Debido a los problemas ambientales que pueden ser causados por la disposición del lodo residual en el medio ambiente, este trabajo tiene como objetivo evaluar su utilización como fertilizante en la especie forestal de eucalipto.

\section{Lodo residual}

El lodo residual posee en su composición nutrientes y elementos que aportan beneficios para el desarrollo de las plantas y que en su mayoría están en forma orgánica; De esta forma, esos elementos son liberados lentamente en el suelo, disminuyendo el riesgo de contaminación ambiental. Además del beneficio nutricional, el lodo aumenta el contenido de materia orgánica y la CIC del suelo (Malta, 2001). En función de su concentración de materia orgánica, carbono y nutrientes, el lodo se ha considerado útil para uso agrícola, debido principalmente a su bajo costo de obtención. De esta manera, según Bettiol y Camargo (2000) el material puede ser recomendado para fertilización en áreas reforestadas como mejorador del suelo o fertilizante. Debido a su alto contenido de materia orgánica, el lodo residual aumenta la retención de agua y la resistencia a la erosión en el suelo (Shiralipour et al., 1992); mejora las condiciones químicas y biológicas del suelo, aumentando la disponibilidad de nutrientes para las plantas $(\mathrm{He}$ et al., 1992).

El lodo residual posee altos niveles de nitrógeno, pudiendo este, ser un factor limitante para su utilización para fines agrícolas o forestales. Para evitar la contaminación del manto freático por la lixiviación de ese nutriente, deberá ser definida la cantidad máxima de aplicación (Bettiol y Camargo, 2000). El lodo es fuente de fósforo, proveniente en gran parte de los detergentes vertidos, los polifosfatos. La utilización de este residuo en el suelo, aumenta la disponibilidad del elemento en las capas superiores, teniendo efecto gradual y residual en el suelo, colaborando para la asimilación del nutriente por las plantas (Ayuso et al., 1992). En relación al potasio, tendrá que ser realizada la adición del mismo cuando exista baja disponibilidad en el suelo (Oliveira et al., 1995).

El lodo residual contiene metales pesados como cobre, fierro, níquel y zinc (Mattiazzo y Andrade, 2000). Este factor causa preocupación para su utilización como fertilizante. Sin embargo, investigaciones demuestran que la utilización del residuo en suelos brasileños no tiene efecto tóxico para las plantas y/o para el suelo (Malta, 2001).

\section{El eucalipto en brasil}

Las primeras plantaciones de eucalipto en Brasil fueron realizadas en 1904, por el Ing. Navarro de Andrade, en el Estado de São Paulo, siendo que él fue el primer autor de libros sobre ese género en Brasil. Inicialmente, la producción de madera era para fabricación de traviesas ferroviarias y leña para las locomotoras que se desplazaban en las vías de hierro del Estado de São Paulo (Wilcken et al., 2008).

En Brasil las especies forestales exóticas, en especial el eucalipto y el pino, son las más plantadas. El eucalipto posee mayor relevancia debido al aumento de las investigaciones y avances tecnológicos en el sector productivo. Este hecho ubica a Brasil entre los países productores tradicionales, siendo el que más 
produce por hectárea/año (Valverde et al., 2012). Aproximadamente 700 especies de eucalipto poseen características de crecimiento rápido, excelente productividad y buena adaptación en varios tipos de clima y suelo. Las principales especies plantadas en Brasil son los híbridos de E. grandis cruzadas con E. urophylla (E. urograndis), E. grandis, E. urophylla, E. saligna, E. viminalis, E. globulus, E. camaldulensis, entre otras (IPEF, 2005).

El eucalipto tiene una gran importancia para la economía brasileña (Tabla 1), gracias al aumento significativo en la plantación de esta especie en las tres últimas décadas, debido al mejoramiento de las prácticas de silvicultura y propagación de clones (Binkley y Stape, 2004). El estado de Minas Gerais posee la mayor área plantada de eucalipto en territorio Brasileño (Figura 1), seguido por São Paulo. Las plantaciones de eucalipto aumentaron $2,4 \%$ en los últimos años, siendo el estado de Mato Grosso del Sur responsable por esa expansión, con 400 mil nuevas hectáreas plantadas (IBÁ, 2017).

Actualmente, la producción de eucalipto presenta el mejor costo-beneficio, pues las plantaciones son de ciclos relativamente cortos en comparación con las demás especies forestales nativas, además de que presenta baja exigencia nutricional. Los bosques de

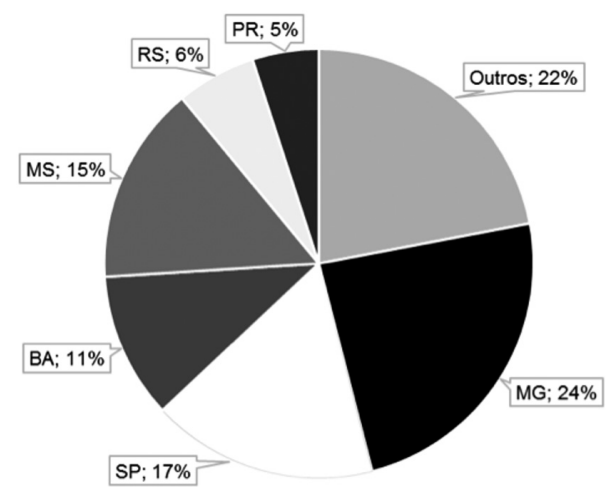

Figura 1. Distribución de las plantaciones de eucalipto por estado en territorio Brasileño en 2016.

Fuente: Adaptado de IBÁ y POYRY (2016).

eucalipto proveen no solo la madera, sino también varios productos (Tabla 2), tales como celulosa, papel, generación de energía y hasta productos fitoterapéuticos (ABAF, 2016).

\section{Resultados de diferentes investigaciones (2008-2018)}

El eucalipto es una especie forestal poco exigente en términos nutricionales, adaptándose

Tabla 1. Clasificación de especies de eucalipto en función de su uso.

\begin{tabular}{|c|c|}
\hline Finalidad & Especies de eucalipto utilizadas \\
\hline Celulosa & $\begin{array}{l}\text { E. alba, E. dunnii, E. globulus, E. grandis, E. saligna, E. urophylla e E. grandis } x \text { E. urophylla e } \\
\text { E. urograndis (híbrido). }\end{array}$ \\
\hline Leña y carbón & $\begin{array}{l}\text { E. brassiana, E. camaldulensis, E. cloeziana, E. crebra, E. deglupta, E. exserta, E. globulus, E. grandis, } \\
\text { E. maculata, E. paniculata, E. pellita, E. pilularis, E. saligna, E. tereticornis, E. tesselaris e E. urophylla. }\end{array}$ \\
\hline Muebles y aserradero & $\begin{array}{l}\text { E. camaldulensis, E. citriodora, E. cloeziana, E. dunnii, E. deglupta, E. exserta, E. globulus, E. grandis, } \\
\text { E. maculata, E. maidenii, E. microcorys, E. paniculata, E. pilularis, E. propinqua, E. punctata, E. resinifera, } \\
\text { E. robusta, E. saligna, E. tereticornis e E. urophylla. }\end{array}$ \\
\hline Laminados & $\begin{array}{l}\text { E. botryoides, E. dunnii, E. grandis, E. maculata, E. microcorys, E. pilularis, E. robusta, E. saligna e } \\
\text { E. tereticornis. }\end{array}$ \\
\hline Fabricación de cajas & E. dunnii, E. grandis, E. pilularis e E. resinifera. \\
\hline Construcción civil & $\begin{array}{l}\text { E. alba, E. botryoides, E. camaldulensis, E. citriodora, E. cloeziana, E. deglupta, E. maculata, E. microcorys, } \\
\text { E. paniculata, E. pilularis, E. resinifera, E. robusta, E. tereticornis e E. tesselaris. }\end{array}$ \\
\hline Traviesas ferroviarias & $\begin{array}{l}\text { E. botryoides, E. camaldulensis, E. cloeziana, E. crebra, E. deglupta, E. exserta, E. maculata, E. maidenii, } \\
\text { E. microcorys, E. paniculata, E. pilularis, E. propinqua, E. punctata, E. robusta e E. tereticornis. }\end{array}$ \\
\hline Postes & $\begin{array}{l}\text { E. camaldulensis, E. cloeziana, E. maculata, E. maidenii, E. microcorys, E. paniculata, E. pilularis, } \\
\text { E. punctata, E. propinqua, E. tereticornis e E. resinifera. }\end{array}$ \\
\hline Aceites esenciales & E. camaldulensis, E. exserta, E. globulus, E. smithii e E. tereticornis. \\
\hline Taninos & E. camaldulensis, E. maculata, E. paniculata e E. smithii. \\
\hline
\end{tabular}

Fuente: Adaptado de IPEF (2005). 
Tabla 2. Consumo de madera de eucalipto para uso industrial en Brasil, año 2016.

\begin{tabular}{lc}
\hline Finalidad & Millones de $\mathrm{m}^{3}$ \\
\hline Celulosa y papel & 70,74 \\
Paneles reconstituidos & 5,93 \\
Industria maderera & 5,86 \\
Carbón & 21,46 \\
Leña industrial & 46,94 \\
Madera tratada & 1,46 \\
Otros usos & 1,57 \\
Total & 153,96 \\
\hline
\end{tabular}

Fuente: Adaptado de IBÁ y POYRY (2016).

bien a suelos ácidos y pobres en nutrientes. Sin embargo, es necesario realizar fertilización en la fase de plántula, con la finalidad de que los arboles crezcan rápidamente en campo. Existen diferentes investigaciones con el uso de lodo residual para fines forestales, desde la producción de plántulas hasta la plantación y crecimiento en campo. De forma general, el contenido de nutrientes en el lodo residual varía dependiendo del lugar en que fue generado. El residuo regularmente es pobre en potasio, siendo que este nutriente posee alta solubilidad en agua y dependiendo del cultivo es necesario adicionar este nutriente en la fertilización, como es el caso del eucalipto.

Plántulas de Eucalyptus grandis, fueron cultivadas en substrato suplementado con volúmenes de $0 ; 25 ; 50 ; 75$ e 100 t ha $^{-1}$ de lodo residual, durante un periodo de 180 días. En este estudio, se observó aumento del área foliar, masa seca total, relación del área foliar, tasas de crecimiento relativo y absoluto de las plántulas. Estos resultados confirman los beneficios del uso del lodo para la producción de plántulas de dicha especie (Garcia et al., 2010).

En otro experimento, plántulas de Eucalyptus citriodora con $15 \mathrm{~cm}$ de altura fueron cultivadas en suelo latosol rojo distro-férrico suplementado con volúmenes entre 10 y 40 t ha $^{-1}$ de lodo residual. Después de 180 días del trasplante para vasos, el tratamiento con 30 t ha $^{-1}$ de lodo residual presentó el mejor resultado para las variables altura, diámetro del tallo y masa seca de la parte aérea. La eficiencia nutricional de las plántulas que recibieron los tratamientos con 30 y $40 \mathrm{t} \mathrm{ha}^{-1}$ de lodo residual, presentaron los valores más altos en comparación con los otros tratamientos. Usando proporciones $25 \%, 50 \%$ e $75 \%$ del compuesto de lodo residual en plántulas de Eucalyptus urograndis, el crecimiento inicial fue satisfactorio, en comparación con el tratamiento de fertilización convencional, siendo la masa seca superior con el tratamiento de 50\% del compuesto adicionado al suelo (Afáz et al., 2017). En otro experimento con plántulas de Eucalyptus camaldulensis de aproximadamente un año fueron aplicadas dosis de 20, 40 e 60\% de lodo residual mezclado con el suelo. Los resultados del experimento demostraron que el tratamiento del $60 \%$ de lodo residual incorporado al suelo, proporcionó aumento del $20 \%$ en el crecimiento de las plántulas y $40 \%$ más en el número de hojas (Leila et al., 2017).

Después de 18 meses de aplicación de lodo residual en Eucalyptus grandis en la etapa vegetativa, los arboles tratados presentaron valores significativos en volumen de madera, en comparación al tratamiento control y valores significativamente iguales al tratamiento con fertilización mineral convencional. Dichos tratamientos fueron 10, 20 y 30 t ha $^{-1}$ con complementación de $\mathrm{K}$ y B debido al bajo contenido de estos nutrientes en el lodo residual (Silva et al., 2008).

Trabajando con suelo Latosol Rojo degradado con una plantación de Eucalyptus citriodora fueron aplicados tratamientos con 30 y $60 \mathrm{t} \mathrm{ha}^{-1}$ de lodo residual. Los resultados indicaron recuperación en las propiedades físicas del suelo después de la aplicación de los tratamientos con lodo, con mejoría en la densidad, microporosidad y porosidad total, sugiriendo efecto de recuperación del suelo. La dosis de $60 \mathrm{t} \mathrm{ha}^{-1}$ fue la que promovió la mayor tasa de crecimiento, en plantas de Eucalyptus citriodora (Campos y Alves, 2008). Aplicando dosis de hasta $40 \mathrm{t} \mathrm{ha}^{-1}$ de lodo residual suplementado con $\mathrm{K}$, en Latosol Rojo-Amarillo con textura media-arenosa, en plantación de Eucalyptus grandis, hubo aumento de la biomasa aérea de los árboles, siendo que la dosis con $10 \mathrm{t} \mathrm{ha} \mathrm{h}^{-1}$, presentó crecimiento del $63 \%$ más que el control. En este experimento, una vez más fue comprobado que el lodo puede suplir gran parte de las demandas nutricionales del eucalipto, contribuyendo para el acumulo de la biomasa en leña que es utilizada en gran escala para la producción comercial de madera (Lira et al., 2008). Analizando la densidad básica de la madera en arboles de Eucaliptus grandis con 18 meses de edad, fueron aplicados tres tratamientos divididos en control, fertilización convencional y $10 \mathrm{t} \mathrm{ha}^{-1}$ de lodo residual suplementado con $\mathrm{K}$. Los resultados mostraron que la densidad básica 
media de leña fue significativa con la aplicación del tratamiento con lodo residual, siendo la más alta con $0,41 \mathrm{~g} \mathrm{~cm}^{-3}$ (Sette Junior et al., 2009).

\section{Conclusiones}

La utilización de lodo residual es técnicamente viable como fertilizante en plantaciones de eucalipto, siendo necesaria la fertilización adicional solamente en algunos casos, dependiendo del origen del residuo y de la disponibilidad de nutrientes en el suelo. El lodo residual presenta efectos positivos para el cultivo de eucalipto, contribuyendo en altura, diámetro de tallo, densidad de la madera y biomasa. El residuo aumenta la materia orgánica del suelo y el nivel de fertilidad, habiendo mayor disponibilidad de nutrientes para las plantas, principalmente $\mathrm{N}$ y $\mathrm{P}$, elevando la productividad del eucalipto. El lodo residual puede ser utilizado para recuperación de suelos degradados, debido a sus efectos positivos en el suelo, mejorando la densidad, porosidad total y macroporosidad. Con relación a los metales pesados presentes en el lodo residual, los diferentes estudios indican que no existe toxicidad en las plantas; sin embargo, debe haber monitoreo continuo y acatar la legislación vigente para no exceder la cantidad establecida por la misma.

\section{Literatura Citada}

ABAF

Anuário Brasileiro da Silvicultura 2016. 2016. Editora Gazeta Santa Cruz. Santa Cruz do Sul, Brasil. 56 p.

Afáz, D.C.S.; Bertolaz, K.B.; Viani, R.A.G.; Souza, C.F.

2017. Composto de lodo de esgoto para o cultivo inicial de eucalipto. Ambiente \& Água-An Interdisciplinary Journal of Applied Science, 12: 112-123.

Andrade, T.C.O.; Broetto, F.; Zabotto, A.R.; Kühn, I.E.; Oliveira, D.P.F.; Ruiz, A.M.M.; Souza, M.L.C.

2018. Lodo de esgoto: contextualização econômico-financeira sobre o uso do lodo de esgoto na agricultura. In: Ramos, E.G.; Zuñiga, E.A.; Machuca, L.M. Estresse das plantas cultivadas \& protocolos de análise. Botucatu: FEPAF. pp. 36-41.

Ayuso, M.; Hernández, T.; García, C.; Costa, F.

1992. Utilización de un lodo aerobio como sustitutivo de fertilizantes fosforados inorgánicos. Suelo y Planta, 2: 271-280.

Bettiol, W.; De Camargo, O.A.

2005. Impacto ambiental do uso agrícola do lodo de esgoto. In: Seminário Internacional Sobre Microbiologia Aplicada ao Meio Ambiente: Antecedentes Históricos e Perspectivas. USP. São Paulo, Brasil. pp. 1-19.

Binkley, D.; Stape, J.L.

2004. Sustainable management of eucalypt plantations in a changing world. In: Tomé, M. (ed). IUFRO Conference: Eucalyptus in a Changing World. Aveiro: RAIZ, Instituto Investigação de Floresta e Papel. Aveiro, Portugal. Pp. 11-15.

Campos, F.S.; Alves, M.C.

2008. Uso de lodo de esgoto na reestruturação de solo degradado. Revista Brasileira de Ciência do Solo, 32: 1389-1397.

Clarke, B.O.; Smith, S.R.

2011. Review of 'emerging'organic contaminants in biosolids and assessment of international research priorities for the agricultural use of biosolids. Environment International, 37: 226-247.

Garcia, G.O.; Gonçalves, I.Z.; Madalão, J.C.; Nazário, A.A.; Dos Reis, E.F.

2010. Crescimento de mudas de eucalipto submetidas à aplicação de biossólidos. Revista Ciência Agronômica, 41: 87-94.
He, X.T.; Traina, S.J.; Logan, T.J.

1992. Chemical properties of municipal solid waste composts. Journal of Environmental Quality, 21: 318-329.

Indústria Brasileira de Árvores. 2017. Relatório 2017. IBA. Sao Paulo, Brasil. 77 p.

Instituto de Pesquisas e Estudos Florestais.

2005. Indicações para escolha de espécies de Eucalyptus. IPEF. Piracicaba, SP - Brasil. Disponible en: http://www. ipef.br/identificacao/eucalyptus/indicacoes.asp. Consultado: 01/ago/ 2018

Leila, S.; Mhamed, M.; Hermann, H.; Mykola, K.; Oliver, W.;

Christin, M.; Nadia, B.

2017. Fertilization value of municipal sewage sludge for Eucalyptus camaldulensis plants. Biotechnology Reports, 13: 8-12.

Lira, A.C.S.D.; Guedes, M.C.; Schalch, V.

2008. Reciclagem de lodo de esgoto em plantação de eucalipto: carbono e nitrogênio. Engenharia Sanitária e Ambiental, 13: $207-216$.

Malta, T.S.

2001. Aplicação de Lodos de Estações de Tratamento de Esgotos na Agricultura: estudo do caso do município de Rio das Ostras-RJ. Fundação Oswaldo Cruz, Escola Nacional de Saúde Pública. Rio de Janeiro, Brazil. 57 p.

Mattiazzo, M.E.; Andrade, C.

2000. Aplicabilidade do biossólido em plantações florestais: IV. Lixiviação de $\mathrm{N}$ inorgânico e toxicidade de metais pesados. In: Bettiol, W.; Camargo, O.A. Impacto ambiental do uso agrícola do lodo de esgoto. Embrapa Meio Ambiente. Jaguariúna, Brazil. pp. 203-207.

Oliveira, D.P.F.; Ruiz, A.M.M.; Broetto, F.; Zabotto, A.R.; Kühn, I.E.; Andrade, T.C.O.; Machuca, L.M.R.

2018. Lodo de esgoto: uso do lodo de esgoto em plantas. In: Ramos, E.G.; Zuñiga, E.A.; Machuca, L.M. Estresse das plantas cultivadas \& protocolos de análise. FEPAF. Botucatu, Brazil. Pp. 51-58.

Oliveira, F.C.; Marques, M.O.; Bellingieri, P.A.; Perecin, D. 1995. Lodo de esgoto como fonte de macronutrientes para a cultura do sorgo granífero. Scientia Agricola, 52: 360-367. 
Poggiani, F.; Silva, P.

2005. Biossólido aumenta produtividade de eucaliptos. Revista Visão Agrícola, 4: 105-107.

Sette Junior, C.R.; Tomazello Filho, M.; Lousada, J.L.; Laclau, J.P. 2009. Efeito da aplicação de fertilização nitrogenada e lodo de esgoto nas características da madeira juvenil de árvores de Eucalyptus grandis. Revista Cerne, 15: 303-312.

Shiralipour, A.; Mcconnell, D.B.; Smith, W.H.

1992. Physical and chemical properties of soils as affected by municipal solid waste compost application. Biomass and Bioenergy, 3: 261-266.

Silva, P.H.M.; Poggiani, F.; Moraes Gonçalves, J.L.D.; Stape, J.L. 2008. Volume de madeira e concentração foliar de nutrientes em parcelas experimentais de Eucalyptus grandis fertilizadas com lodos de esgoto úmido e seco. Revista Árvore, 32: 845-854.
Valverde, S. R.; Antunes Mafra, J. W.; Miranda, M. A. da; Silva Souza, C.; Campos Vasconcelos, D.

2012. Silvicultura Brasileira - Oportunidades e desafios da economia verde. Fundação Brasileira para o Desenvolvimento Sustentável. Rio de Janeiro, RJ, Brasil. 39 p.

Wilcken, C.F.;Lima, A.C.V.; Dias, T.K.R.; Masson, M.V.; Ferreira Filho, P.J.; Pogetto, M.H.F.A.

2008. Guia prático de manejo de plantações de eucalipto. FEPAF. Botucatu, Brazil. 19 p.

Zabotto, A.R.; Kühn, I.E.; Broetto, F.; Oliveira, D.P.F.; Ruiz, A.M.M.; Andrade, T.C.O.; Zuñiga, E.A.

2018. Lodo de esgoto: características nutricionais e efeitos da utilização no solo. In: Ramos, E.G.; Zuñiga, E.A.; Machuca, L.M. Estresse das plantas cultivadas \& protocolos de análise. FEPAF. Botucatu, Brazil. pp. 42-50. 